Pacific Journal of Mathematic 


\title{
SUMS OF SQUARES IN PLANAR NASH RINGS
}

\author{
Gustave A. EFroymson
}

\begin{abstract}
In a previous paper, the author developed the ideal theory of Nash rings on planar domains. Here that theory is applied to show that a Nash function on a planar domain $D$ which takes on only non-negative values on $D$ is a sum of two squares of Nash functions on $D$. Examples are given which clarify the situation for higher dimensions.
\end{abstract}

o. Introduction. In the paper, "Nash Rings on Planar Domains" [2], the ideal structure of planar Nash rings was discussed. Using the results of this paper, it is possible to show that for $D$ a planar semi-algebraic domain, that if $f \in N_{D}$, the Nash ring on $D$, then $f \geqq 0$ on $D$ implies that there exist $f_{1}, f_{2}$ in $N_{D}$ with $f=f_{1}^{2}+f_{2}^{2}$. Recall that for polynomials, i.e. for $R[x, y]$, it is known that there exist polynomials $p(x, y)$ for which there is no representation as a sum of any number of squares of polynomials (e.g. Motzkin's example [3]) and that if we use elements in $R(x, y)$ four squares may be necessary [1]. As can be seen, the situation for Nash rings is merely shifted by one dimension since by "homogenizing" Motzkin's example, one obtains a Nash function which can't be represented as a sum of squares of any number of Nash functions, and if quotients of Nash functions are allowed, at least four are necessary to get this function as a sum of squares.

1. The Main Theorem. We first recall some definitions.

DEFINITION 1. A semi-algebraic planar domain is a semi-algebraic domain $D \subseteq R^{2}$. In addition to $D$ being open, and connected, we require that $D$ can be defined by a finite number of polynomial inequalities.

Definition 2. A Nash function on $D$ is a real valued function $f(x, y)$ such that $f$ is analytic on $D$, and there exists a polynomial $p_{f}(x, y, z)$ such that $p_{f}(x, y, f(x, y))=0$ identically on $D$. This is just to say $f$ is algebraic and analytic on $D$.

Definition 3. For $D$ as above, $N_{D}=$ the ring of Nash functions on $D$.

The main result of this paper can now be stated.

ThEOREM 1. Let $N_{D}$ be as above and suppose $f$ is an element of 
$N_{D}$ such that $f \geqq 0$ on $D$. Then there exist $f_{1}$ and $f_{2}$ in $N_{D}$ so that $f=f_{1}^{2}+f_{2}^{2}$.

Recall that for analytic functions, one has the exact sequence of sheaves of germs of analytic functions:

$$
0 \longrightarrow \mathcal{O}_{X} \stackrel{f \rightarrow e^{f}}{\longrightarrow} \mathcal{O}_{X}^{*} \longrightarrow Z_{2} \longrightarrow 0 \text {. }
$$

Here $\mathscr{O}_{X}$ is the sheaf of germs of analytic functions on $X$, and $\mathscr{O}_{X}^{*}$ is the sheaf of non-zero germs under multiplication. From the long exact sequence for cohomology, one finds:

$$
H^{1}\left(X, \mathscr{O}_{X}\right) \longrightarrow H^{1}\left(X, \mathscr{O}_{X}^{*}\right) \longrightarrow H^{1}\left(X, Z_{2}\right) \longrightarrow H^{2}\left(X, \mathscr{O}_{X}\right)
$$

but since $H^{1}\left(X, \mathscr{O}_{X}\right)=H^{2}\left(X, \mathscr{O}_{X}\right)=0$, we obtain $H^{1}\left(X, \mathscr{O}_{X}^{*}\right) \cong H^{1}\left(X, Z_{2}\right)$. We also have the exact sequence of sheaves:

$$
1 \longrightarrow \mathscr{O}_{*}^{X} \longrightarrow \mathscr{H}^{*} \longrightarrow \mathscr{H}^{*} \mid \mathscr{O}_{X}^{*}=\mathscr{D} \longrightarrow 1
$$

where $\mathscr{C}^{*}$ is the sheaf of germs of meromorphic functions and $D$ is the sheaf of divisors. Again the long exact sequence can be taken

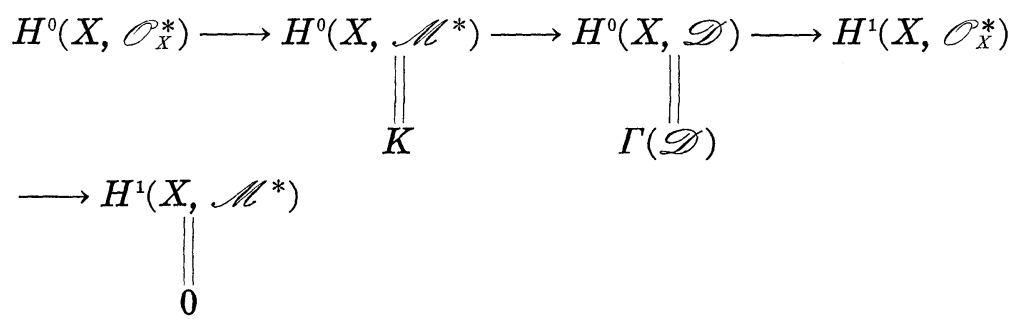

so $H^{1}\left(X, Z_{2}\right) \cong H^{1}\left(X, \mathscr{O}_{X}^{*}\right) \cong \Gamma(\mathscr{D}) / K=$ class group of $\Gamma\left(\mathscr{O}_{X}\right)$.

If $D$ is a simply connected region in $R^{2}$, we find $H^{1}\left(D, Z_{2}\right)=0$. This implies by the above result that the class group is trivial. In [2] we showed that if $H^{1}\left(D, Z_{2}\right)=0$, then the class group for $N_{D}$, the ring of Nash functions on $D$, is also trivial even though one no longer has $H^{1}\left(X, N_{D}\right)=0$. This means that to give a Nash divisor (locally) on $R^{2}$ is to define up to a unit a Nash function which will generate the principal ideal so defined. Also, since every Nash function only vanishes on parts of algebraic sets which therefore must be unions of analytic components of algebraic sets, we see that the support of a Nash divisor on $N_{D}$ will be a union of analytically connected components of an algebraic set. A minimal such set will be either a curve $C$ or a real point $P$. We call the associated prime $\mathscr{P}_{C}$ or $\mathscr{P}_{P}$. In the case of a point there is some ambiguity since the same point may be the support of different primes; for example, $\left(x^{2}+y^{2}\right)$ and $\left(x^{2}+2 y^{2}\right)$ are different primes with support at the origin. Of course, the complex support is much larger. 
For general semi-algebraic regions $D \subset R^{2}$, there will be analytically connected arcs of algebraic curves which run from one hole of $D$ to another. So we have 3 possibilities for irreducible primes in $N_{D}$. First we have those primes $\mathscr{P}_{C}$ which have support a curve $C$ which passes through no hole of $D$. For such a prime, there exists a global Nash function $f$ on $R^{2}$ with $(f)=\mathscr{P}_{c}$. Then there are the primes associated with a curve running from one hole of $D$ to another, and the third possibility is a prime with real support on one point.

Now take a function $f \in N_{D}$. Then $(f)=\prod_{i, j} \mathscr{P}_{c_{i}} \mathscr{P}_{P_{j}}$. We claim that each $\mathscr{P}_{C_{i}}$ must occur to an even power if $f \geqq 0$ on $D$. For, let $t$ be a local parameter for $\mathscr{P}_{C_{i}}$ at a non-singular point $P$ of $C_{i}$ where no other divisor of $f$ has support. Then if $f=h t^{2 m+1}$ locally, where $h$ is a unit at $P$, we would find that $f$ changes sign near $P$ and so we would get a contradiction.

So if $C_{i}$ is of the first type, $\mathscr{P}_{C_{i}}$ is principal and $=\left(f_{i}\right)$, and so $f_{i}^{2 m}$ divides $f$ for some integer $m$. And if $C_{i}$ is of the second type, $\mathscr{P}_{C_{i}}^{2}$ again divides $(f)$, but $\mathscr{P}_{C_{i}}^{2}$ is principal since the class group is $H^{1}\left(D, Z_{2}\right)$ as shown in [2], and so $\mathscr{P}_{C_{i}}^{2}=\left(g_{i}\right)$ and $g_{i}^{m}$ will divide $f$ for some integer $m$. We now have reduced the proof of Theorem 1 to the following cases, using the fact that if $f_{1}$ and $f_{2}$ are sums of two squares, then so is $f_{1} f_{2}$.

Case 1. The support of $f$ is one point and $f$ is irreducible in $N_{D}$. There exists an irreducible polynomial $p(x, y)$ such that $f$ divides $p$ in $N_{D}$. We can assume that the support of $f$ is the point $(0,0)$, using translation. It was shown in [2] that, by a change of variables, one can get $p$ so that it factors $p=\Pi_{j}\left(y-\alpha_{j}(x)\right)$ and each $\alpha_{j}(x)$ is analytic except possibly at $x=0$. The factors which divide $f$ at $(0,0)$ are thus only those for which $\alpha_{j}(0)=0$. By multiplying the factors which divide $f$ in a complex neighborhood of $(0,0)$, we get $f$ back, up to a factor which is a unit at $(0,0)$. Note also that since $f$ is real, if $y-\alpha_{j}(x)$ is a factor of $f$ so is $y-\bar{\alpha}_{j}$ the complex conjugate.

Now expand $\alpha_{j}(x)$ in Puiseux series and get $\sum a_{k} x^{k}, k \in Q$, with bounded denominators. Suppose all these are in $C\left(\left(x^{1} / n\right)\right)$. Then, since the Galois group of $C\left(\left(x^{1 / n}\right)\right) / C((x))$ is generated by the substitution $x^{1 / n} \rightarrow \omega_{n} x^{1 / n}$, for $\omega_{n}$ a primitive $n$th root of unity, we see that the conjugates of $y_{1}=\sum a_{k} x^{k}$ are just $y_{j}=\sum a_{k} \omega_{n}^{k j} x^{k}$, (over $\boldsymbol{C}((x)), j=$ $1, \cdots, n)$. As long as no two of the $y_{j}$ are complex conjugates, we are done since after multiplying together all the $\left(y-y_{j}\right)$, we will get a function $h(x, y)$ in $N_{D}[i]$. So $h \bar{h}$ will be a sum of two squares in $N_{D}$.

So now suppose two of the $y_{j}$ are complex conjugates. We can 
suppose one is $y_{1}$ and reparameterizing by letting $x=t^{n}$, we obtain $y_{1}=\sum_{i=1}^{\infty} a_{i} t^{i}$. So suppose $y_{k}=\bar{y}_{1}=\sum \bar{a}_{j} t^{j}=\sum a_{j} \omega^{j k} t^{j}, a_{j}=R_{j} e^{i \theta_{j}}$ and $\omega=e^{i \alpha}$. Then $\bar{a}_{j}=R_{j} \bar{e}^{i \theta_{j}}=R_{j} e^{i \theta_{j}} e^{i \alpha j}$. From this it follows that $j \alpha+\theta_{j} \equiv-\theta_{j} \bmod 2 \pi$ and so $\theta_{j} \equiv-j(\alpha / 2) \bmod \pi$. Let $u=e^{-(i \alpha / 2)} t$ and $\sum a_{j} t^{j}=\sum a_{j} e^{i(\alpha j / 2)} u^{j}=\sum \varepsilon_{j} R_{j} u^{j}$ where $\varepsilon_{j}= \pm 1$ and then $x= \pm u^{n}$. Now we have a real parameterization of the curve and so don't have an isolated point.

Case 2. The support of $f$ is an arc $C$ which runs from one hole of $D$ to another. Next, we send one end of $C$ to infinity and the other end to $(0,0)$. Then find half lines $L_{1}, L_{2}$ going from $(0,0)$ to infinity so that $L_{1} \neq L_{2}$ and neither $L_{1}$ nor $L_{2}$ passes through any singular point of $C$ in $D$. Let $V_{i}=L_{i} \cup C$. Now $I\left(L_{i} \cup C\right)=I\left(L_{i}\right) I(C)$. By the Corollary on p. 444 of [2], we find that if the $C_{i}$ are arcs which run from one hole of $D$ to another, then $\prod_{i} I\left(C_{i}\right)$ is principal iff the number of ends of the $C_{i}$ at each hole is even. This is, of course, just another way of saying that the class group of $N_{D}$ is isomorphic to $H^{1}\left(D, Z_{2}\right)$. So since both $L_{i}$ and $C$ have one end at $(0,0)$ and the other at infinity, the above result implies that $I\left(L_{i} \cup C\right)$ is principal. So there exists $f_{i}$ in $N_{D}$ with $I\left(V_{i}\right)=\left(f_{i}\right)$. Now $I$ claim that $f /\left(f_{1}^{n}+f_{2}^{2}\right)$ is a unit in $N_{D}$. Just look locally at each $P$ of $D$. We only have to worry about $P$ on $C$ and then have two subcases. First, when $P$ lies on $C$ but not on $L_{1}$ or $L_{2}$, then the fact that $f$ vanishes on $C$ implies that $f$ is a multiple (locally) of $f_{i}, i=1,2$. But $f=u f_{i}$, for $u$ a local unit, is impossible since $f$ doesn't change sign near $P$. So $f=g_{i} f_{i}^{2}$ for some $g_{i}$, again locally at $P$. Now, since $P$ is not on $L_{1}$ or $L_{2}$, each $f_{i}$ locally generates $I(C)$, so $f_{1}=u f_{2}$ for $u$ unit at $P$. So $f_{1}^{2}+f_{2}^{2}=\left(1+u^{2}\right) f_{2}^{2}$ and so divides $f$ at $P$. Now assume that $P$ is at an intersection of $L_{1}$ and $C$ (not the origin which isn't even in $D$ ), then $C$ is non-singular at $P$ and we can assume the intersection of $L_{1}$ and $C$ is transversal (moving $L_{1}$ a little if necessary). Now let $t_{C}$ and $t_{L_{1}}$ be local parameters for $C$ and $L_{1}$ respectively. Then $f_{1}=t_{c} t_{L_{1}} u_{1}, f_{2}=t_{C} u_{2}$, for $u_{1}$ and $u_{2}$ units at $P$. So we see $f_{1}^{2}+f_{2}^{2}=t_{i}^{2}\left(u_{2}^{2}+u_{1}^{2} t_{L_{1}}^{2}\right)$, which implies that $f_{1}^{2}+f_{2}^{2}$ divides $f$ at $P$. Thus $f / f_{1}^{2}+f_{2}^{2}$ is analytic on $D$. Since $f$ is irreducible, $f=u\left(f_{1}^{2}+f_{2}^{2}\right)$, for $u$ a unit in $N_{D}$. Then $v=u^{1 / 2}$ is in $N_{D}$ and $f=$ $\left(v f_{1}\right)^{2}+\left(v f_{2}\right)^{2}$.

Q.E.D.

\section{An example.}

EXAMPLE. Let $f=x+\left(x^{2}+y^{2}\right)^{1 / 2}$ in $N_{D}$, where $D=R^{2}-(0,0)$. Here $C$ is the negative $y$-axis. We can let $L_{1}$ be the positive $x$-axis and $L_{2}$ be the negative $x$-axis. Using the methods in [2], we find $f_{1}=x-y+\left(x^{2}+y^{2}\right)^{1 / 2}, f_{2}=x+y+\left(x^{2}+y^{2}\right)^{1 / 2}$. So we find $f_{1}^{2}+f_{2}^{2}=$ 
$2\left(x+\left(x^{2}+y^{2}\right)^{1 / 2}\right)^{2}+2 y^{2}=4\left(x^{2}+y^{2}+x\left(x^{2}+y^{2}\right)^{1 / 2}\right)=4\left(x^{2}+y^{2}\right)^{1 / 2}\left(x+\left(x^{2}+\right.\right.$ $\left.\left.y^{2}\right)^{1 / 2}\right)$. This means $v$ should be $2\left(x^{2}+y^{2}\right)^{1 / 4}$ and finally $f_{1}=(x-y+$ $\left.\left(x^{2}+y^{2}\right)^{1 / 2}\right) / 2\left(x^{2}+y^{2}\right)^{1 / 4}, f_{2}=\left(x+y+\left(x^{2}+y^{2}\right)^{1 / 2}\right) / 2\left(x^{2}+y^{2}\right)^{1 / 4}$. Of course other representations are also possible.

3. No higher dimensional result. Finally, consider Motzkin's example, $p(x, y)=1+x^{2}\left(x^{2}-3\right) y^{2}+x^{2} y^{4}$ in $R[x, y]$. Motzkin [3] showed that this polynomial is $\geqq 0$ everywhere but is not the sum any number of squares in $R[x, y]$ or see [4]. Moreover, Cassels, Ellison, and Pfister [1] have shown that $p$ is not the sum of 3 squares in $R(x, y)$. For our purposes, we homogenize $p(x, y)$ to get $q(x, y, z)=$ $z^{6}+x^{2}\left(x^{2}-3 z^{2}\right) y^{2}+x^{2} y^{4}$. Let $D$ be any domain $R^{3}$ containing $(0,0,0)$. We find with the above notation.

THEOREM 2. The homogeneous polynomial $q(x, y, z)$ is not the sum of any number of squares elements of $N_{D}$. Moreover $q$ is not the sum of three squares in the quotient field of $N_{D}$.

Proof. Assume $q=\sum h_{i}^{2}$ where the $h_{i}$ are in $N_{D}$. Since each $h_{i}$ is analytic at $(0,0,0)$ we can expand in power series, and looking at the lowest degree terms which appear in any $h_{i}$ (and which must be of degree 3) we get a new equation $\sum g_{i}^{2}=q(x, y, z)$ homogeneous of degree 6. Divide by $z^{6}$ and dehomogenize and we get a contradiction to Motzkin's result. Finally, let $q=\sum_{i=1}^{3} h_{i}^{2} / g^{2}$ where the $h_{i}$ and $g$ are in $N_{D}$. Then $g^{2} q=\sum h_{i}^{2}$. Again look at the lowest degree terms on each side and we get a homogeneous equation which when dehomogenized gives a contradiction to Cassels, Ellison, and Pfister, loc. cit. One should note that $q \geqq 0$ for all $z, y, z$ which is clear since when $z \neq 0$ we can dehomogenize and when $z=0$, we get $x^{4} y^{2}+x^{2} y^{4}$.

\section{REFERENCES}

1. J. W. S. Cassels, W. J. Ellison and A. Pfister, On sums of squares and elliptic curves over function fields, J. Number Theory, 3 (1971), 125-149.

2. G. Efroymson, Nash rings on planar domains, Trans. Amer. Math. Soc. 249 (1979), 435-445.

3. T.S. Motzkin, The arithmetic-geometric inequality, in "Inequalities" (0. Shisha, editor) pp. 205-224, Academic Press, NY, 1967.

4. A. Pfister, Quadratic forms over fields, Proc. of Symposia in Pure Math. XX, 150160, AMS, Providence, (1971).

Received July 10, 1980.

UNIVERSiTy OF NeW MEXICO

ALBUQUERQUE NM 87131 



\section{PACIFIC JOURNAL OF MATHEMATICS}

\section{EDITORS}

DONALD BABBITT (Managing Editor)

University of California

Los Angeles, California 90024

Hugo RossI

University of Utah

Salt Lake City, UT 84112

C. C. MOORE and ANDREW OGG

University of California

Berkeley, CA 94720

\section{J. DugundJI}

Department of Mathematics University of Southern California Los Angeles, California 90007

R. Finn and J. Milgram Stanford University

Stanford, California 94305

\section{ASSOCIATE EDITORS}
R. ARENS
E. F. BeCKENBACH
B. H. Neumann
F. WoLF
K. YoSHIDA

\section{SUPPORTING INSTITUTIONS}

UNIVERSITY OF ARIZONA

UNIVERSITY OF BRITISH COLUMBIA

CALIFORNIA INSTITUTE OF TECHNOLOGY

UNIVERSITY OF CALIFORNIA

MONTANA STATE UNIVERSITY

UNIVERSITY OF NEVADA, RENO

NEW MEXICO STATE UNIVERSITY

OREGON STATE UNIVERSITY
UNIVERSITY OF OREGON

UNIVERSITY OF SOUTHERN CALIFORNIA

STANFORD UNIVERSITY

UNIVERSITY OF HAWAII

UNIVERSITY OF TOKYO

UNIVERSITY OF UTAH

WASHINGTON STATE UNIVERSITY

UNIVERSITY OF WASHINGTON 


\section{Pacific Journal of Mathematics}

\section{Vol. 97, No. $1 \quad$ January, 1981}

Charles A. Asmuth and Joe Repka, Tensor products for $S L_{2}(\mathfrak{k})$. II.

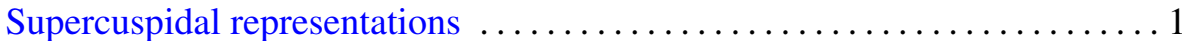

Joseph Barback, On finite sums of regressive isols . ................. 19

Matthew G. Brin and Daniel Russell McMillan, Jr., Generalized

three-manifolds with zero-dimensional nonmanifold set ............29

Kun Soo Chang, Converse measurability theorems for Yeh-Wiener space . . . 59

Christopher Brian Croke, A "maximal torus" type theorem for complete

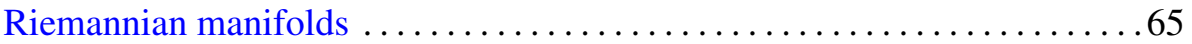

Gustave Adam Efroymson, Sums of squares in planar Nash rings . . . . . . 75

John Robert Fisher, Axiomatic radical and semisimple classes of rings . . . .81

Betty Kvarda, Consecutive integers for which $n^{2}+1$ is composite .......93

Roosevelt Gentry, New diagram proofs of the Hausdorff-Young theorem

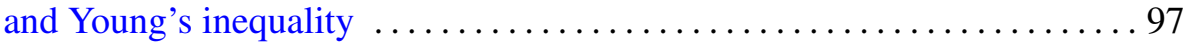

Patrick M. Gilmer, Topological proof of the $G$-signature theorem for $G$

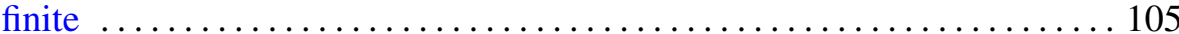

Chung Wei Ha, A noncompact minimax theorem .................. 115

James J. Hebda, Manifolds admitting taut hyperspheres ................ 119

Takayuki Kawada, Sample functions of Pólya processes ............. 125

Peter K. F. Kuhfittig, Common fixed points of nonexpansive mappings by

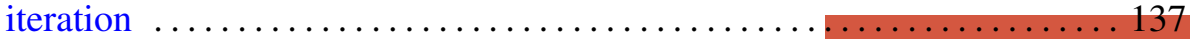

James Thomas Loats and Judith Roitman, Almost rigid Hopfian and dual

Hopfian atomic Boolean algebras .......................... 141

Roger McCann, On embedding semiflows into a radial flow on $l_{2} \ldots \ldots \ldots 151$

John McDonald, Closed orbits of convex sets of operators on the disk

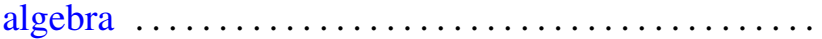

Mark D. Meyerson, Convexity and the table theorem .............. 167

Arnold William Miller, Generic Souslin sets . . . . . . . . . . . . . . . . 171

Takemi Mizokami, On the closed images of paracomplexes $\ldots \ldots \ldots \ldots \ldots 3$

Jagannadham Venkata Pakala and Thomas Stephen Shores, On

compactly packed rings $\ldots \ldots \ldots \ldots \ldots \ldots \ldots \ldots \ldots$

Andrew Pletch, Strong completeness in profinite groups

Wilbur Carrington Whitten, Inverting double knots

James Juei-Chin Yeh, Existence of strong solutions for stochastic

differential equations in the plane 